\title{
FFT Factorization Technique for OFDM System
}

\author{
Tanvi Chawla \\ Haryana College of Technology \& Management, Kaithal, Haryana, India
}

\begin{abstract}
For OFDM communication systems, the frequency offsets in mobile radio channels deteriorate the orthogonality between subcarriers resulting in Inter Carrier Interference (ICI). ICI causes power leakage among subcarriers thus degrading the system performance. A well-known problem of OFDM is its sensitivity to frequency offset between the transmitted and received carrier frequencies. This research work investigates three bandwidth efficient methods for combating the effects of ICI: DFT-OFDM algorithm, two path algorithm (TPA) and these three methods are compared in terms of bit error rate performance with Proposed FFT Factorization Technique. Through simulations, it is shown that proposed Technique is much effective in mitigating ICI at high frequency offset.

Keywords: Self-cancellation,ICI,BER,CIR
\end{abstract}

\section{INTRODUCTION}

Orthogonal frequency division multiplexing has gained considerable attention due to its high speed data applications. It is emerging as most preferred digital modulation technique due to its high bandwidth efficiency and strength to handle multipath interference. OFDM is a multicarrier transmission system in which transmission bandwidth is divided into many narrowband sub channels which are transmitted into parallel. As a result the symbol duration is increased and inter-symbol interference is mitigated. OFDM systems require precise frequency synchronization otherwise inter-carrier interference (ICI) occurs [1]. ICI has a negative impact on data throughput. In literature there are several methods to reduce ICI. The first approach is frequency-domain equalization in [2], in which, weighting coefficients of equalizer need be chosen by channel state information (CSI) estimation. The second one is time-domain windowing in [3]. The third is ICI self-cancellation in [4], which maps the transmitted data onto a group of adjacent subcarriers with a group of weighting coefficient rather than onto single subcarrier, and then the ICI can be reduced by linearly combining the received signals. This method is very simple and significantly provides CIR improvement, however bandwidth efficiency of OFDM system gets reduced.

This paper is focused on ICI-self-cancellation technique. There are various self-cancellation techniques with different ICI cancelling modulation methods and the corresponding demodulation methods, proposed to reduce the ICI caused by carrier frequency offset (CFO). These methods include the adjacent data-conversion method [5], the symmetric dataconversion method [6], the adjacent data-conjugate method, and the symmetric data-conjugate method [7].The aforementioned methods can suppress the ICI effectively, but the spectral efficiency will obviously be reduced as data is replicated on two or more subcarriers.
To increase bandwidth efficiency various schemes has been proposed in literature. Bing Han et al. proposed DFT based bandwidth efficient method for ICI cancellation [8].In this scheme at the transmitter side, before modulated onto a groupof adjacent subcarriers, the parallel data symbols are divided into two groups, each discrete Fourier transformed. In this scheme with additional signal processing the IC1 can be counteracted to some extent. However, this scheme outperforms only for small frequency offset values $(€<0.2)$.

In this direction another scheme based on two path algorithm (TPA) was proposed in [9]. The first path sends a specially modulated data symbols which results from weighted subtraction of an even numbered modulated symbol and its consecutive symbol. The second path uses the conjugate of a similar type of specially modulated data symbols which results from weighted addition of an even numbered modulated symbol and its consecutive symbol.With additional signal processing bandwidth efficiency of system can be achieved.

In this paper, a new bandwidth efficient scheme is proposed in which parallel Fast Fourier Transform (FFT) factorization is performed using Radix -2 DIF FFT algorithms derived from the well known Cooley-turkey factorization using butterfly algorithm. The decimation in frequency (DIF) radix-2 FFT partitions the DFT computations into even indexed and odd indexed outputs, which can be computed by shorter length DFT's of different combinations of input samples. Recursive application of this decomposition to the shorter length DFT results in the full radix DIF-FFT. Paper concentrate on ICI analysis of proposed scheme and also a theoretical CIR expression is derived. The paper is organized as: In section II system model of proposed scheme is presented. Section III, discusses simulation results of proposed scheme and compared with previous bandwidth efficient schemes. Finally, the overall findings of the study are summarized in Section IV.

\section{PROPOSED MODEL}

A new bandwidth efficient scheme is proposed in which parallel Fast Fourier Transform (FFT) factorization is performed using Radix -2 DIF FFT algorithms derived from the well knownCooley-turkey factorization using butterfly algorithm. The decimation in frequency (DIF) radix-2 FFT partitions the DFT computations into even indexed and odd indexed outputs, which can be computed by shorter length DFT's of different combinations of input samples. Recursive application of this decomposition to the shorter length DFT results in the full radix DIF-FFT. The proposed scheme cancels the ICI coefficient and improves system performance. 


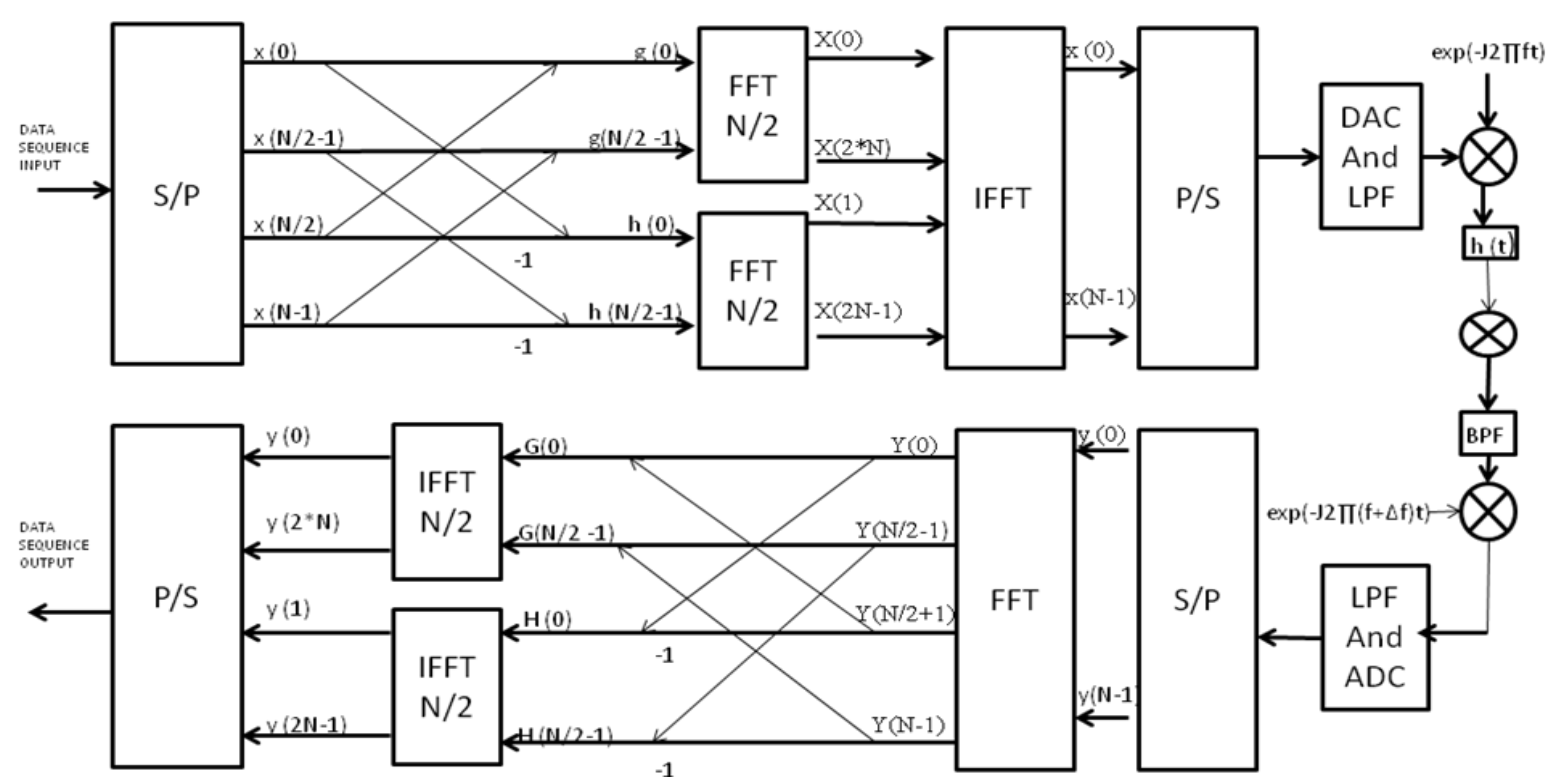

Fig 1: Block diagram of FFT Factorization based ICI self-cancellation technique

\section{Mathematical Description}

In proposed model, $\mathrm{N} / 2$ factorization is shown for the decimation in frequency (DIF) FFT. The sequence of $\mathrm{N}$ complex data symbols $[x(0) \ldots \ldots \ldots x(N-1)]$ (time domain) are transformed into data sequence as $[X(1) \ldots \ldots \ldots . . . .(N-1)]$ by two N/2 FFT (Fast Fourier Transform) blocks as shown in figure 1 . The data sequence of $\mathrm{N}$ points is divided into two equal halves.

The sequence $\mathrm{x}(\mathrm{n})$ which is in time domain after mapping to the subcarrier is taken, then apply an operation of Radix-2 FFT factorization on it

$\mathrm{X}(2 \mathrm{k})=\sum_{n=0}^{\frac{N}{2}-1}\left[x(n)+x\left(n+\frac{N}{2}\right)\right] W_{N / 2}^{n k}(1 \mathrm{a})$

$\mathrm{X}(2 \mathrm{k}+1)=\sum_{n=0}^{\frac{N}{2}-1}\left[x(n)-x\left(n+\frac{N}{2}\right)\right] \mathrm{W}_{\mathrm{N}}^{\mathrm{n}} \mathrm{W}_{\mathrm{N} / 2}^{\mathrm{nk}}(1 \mathrm{~b})$

$X(2 k)$ and $X(2 k+1)$ denote the subsequences of those elements of $X(k)$ that have even or odd indices and then follow the same operation as standard OFDM. IFFT of

$\mathrm{X}(\mathrm{k})$ is

$\mathrm{x}(\mathrm{n})=\frac{1}{N}\left[\sum_{k=0}^{N-1} X(k) W_{N}^{-n k}\right]$

\section{ICI Mechanism}

In the presence of additive white Gaussian noise (AWGN) and carrier frequency offset $(\mathcal{C})$, the received signal on subcarrier can be written as

$$
\mathrm{y}(\mathrm{n})=\quad \sum_{l=0, l \neq k}^{N-1} x(l) * S(l-k)+x(n) S(0)+n_{k}
$$

$(a)(b)(c)$

On right hand side of $(3),(a)$ is desired sequence, $(b)$ is ICI and $(c)$ is AWGN noise introduced in the channel. $x(l)$ is transmitted symbol for the $l^{\text {th }}$ subcarrier and the sequence $\mathrm{S}(1-$ $\mathrm{k})$ is defined as the ICI co-efficient between $l^{\text {th }}$ and $\mathrm{k}^{\text {th }}$ sub carrier which is expressed as

$S(l-k)=\frac{\sin [\pi(l+\varepsilon-k)]}{N \sin \left[\frac{\pi}{N}(l+\varepsilon-k)\right]}$

where $C$ or ep is frequency offset.

\section{ICI Cancelling Demodulation}

Let us continue the derivation for ICI co-efficient only

y $(1)=\sum_{l=0, l \neq k}^{N-1} x(l) * S(l-k)$

At receiver, FFT of it is as

$\mathrm{Y}(\mathrm{k})=\sum_{l \neq k, l=0}^{N-1}[x(l) S(l-k)] W_{N}^{l k}(5 \mathrm{~b})$

Then IFFT

$$
\begin{aligned}
& \begin{array}{l}
\text { y }(2 \mathrm{l})=\frac{1}{N}\left[\sum_{k=0}^{\frac{N}{2}-1}\left[Y(k)+Y\left(k+\frac{N}{2}\right)\right] W_{N / 2}^{-l k}\right. \\
=\frac{1}{N} \sum_{k=0}^{\frac{N}{2}-1} \quad \sum_{l \neq k, l=0}^{N-1}[x(l) * S(l-k)+x(l) *
\end{array} \\
& S(l-k+N / 2)] W_{N / 2}^{-l k} W_{N}^{l k} \\
& =\frac{1}{N} \sum_{k=0}^{\frac{N}{2}-1} \sum_{l \neq k, l=0}^{N-1} x(l)[S(l-k)+S(l-k+ \\
& \left.\left.\frac{N}{2}\right)\right] W_{N}^{-l k}
\end{aligned}
$$


By applying symmetric property $\mathrm{W}_{\mathrm{N}}{ }^{-1 \mathrm{k}}=\mathrm{W}_{\mathrm{N}}{ }^{(\mathrm{k}+\mathrm{N} / 2)}$ and periodicity property results in, For even values of $1, \mathrm{y}(21)=0$ and for odd S (1-k)/N. Similarly for another IFFT N/2 $\mathrm{y}(2 l+1)=\frac{1}{N} \sum_{k=0}^{N-1} \sum_{l \neq k, l=0}^{N-1} x(l)[S(l-k)-S(l-k+$ $\left.\left.\frac{N}{2}\right)\right] W_{N}^{-l k} W_{N}^{-l}(5 \mathrm{~d})$

For even values of $1, \mathrm{y}(21+1)=\mathrm{S}(1-\mathrm{k}) / \mathrm{N}$ and for odd $y(21+1)=0$.So nearly half of ICI coefficients are zero.

The first term in the right-hand side of (3) represents the desired signal. Without frequency error takes its maximum value. The ICI components results from ICI cancelling demodulation mechanism as

$\mathrm{S}^{\prime}(\mathrm{l}-\mathrm{k})^{\mathrm{e}}=\frac{\mathrm{S}(\mathrm{l}-\mathrm{k})+\mathrm{S}\left(\mathrm{l}-\mathrm{k}+\frac{\mathrm{N}}{2}\right)}{\mathrm{N}}$

$S^{\prime}(l-k)^{o}=\frac{S(l-k)-S\left(l-k+\frac{N}{2}\right)}{N}$

The comparison of ICI coefficients of standard model in (4) and of proposed model is in figure 2 under logarithmic scale at $\mathrm{E}=0.2$ and $\mathrm{N}=64$. For

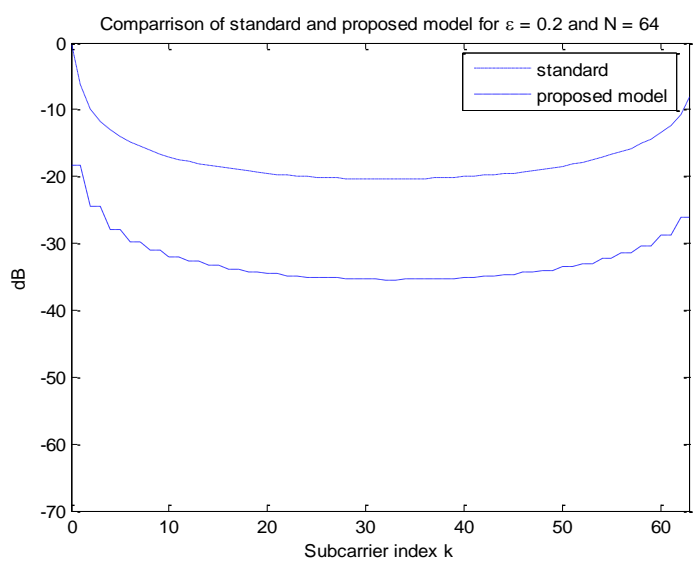

Fig.2: Comparison of S(l-k) of standard and S'(l-k)

$$
\text { for } N=64
$$

most $\mathrm{S}(\mathrm{l}-\mathrm{k})$ values

$$
S^{\prime}(l-k)<<S(l-k)
$$

Figure 3 gives an example of the S' (1-k) which is combined effect of odd and even indices when $\mathrm{l}=0$ and $\mathrm{N}=16$.The frequency offset values are $\epsilon=0.2$ and $\epsilon=0.4$. It is evident that as becomes larger, the desired part decreases and the undesired part increase.
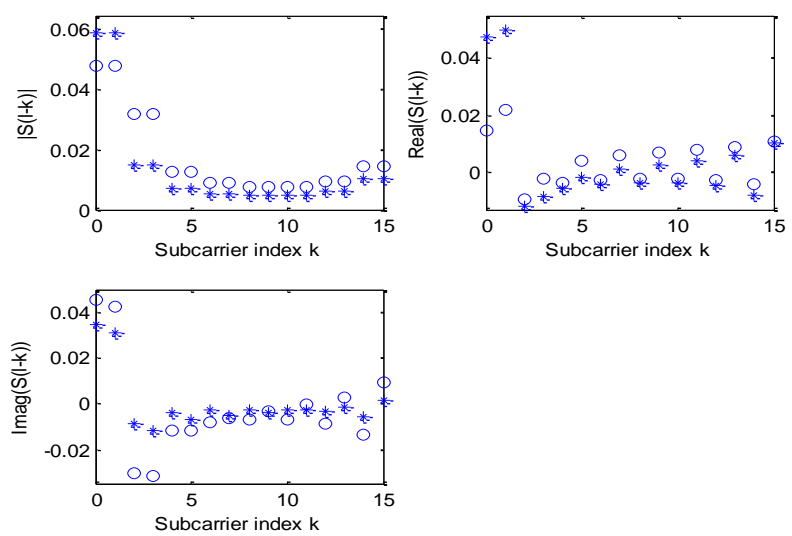

Fig3:- An example of the $S^{\prime}$ (l-k) when $\mathrm{l}=0$ and $\mathrm{N}=16$ with amplitude, real part and Imaginary part of S' (1-k)

The system ICI power level can be evaluated by using the CIR. While deriving the theoretical CIR expression, the additive noise is omitted .CIR is calculated for even and odd separately as per desired or undesired ICI coefficients by equation

$$
\begin{aligned}
& \mathrm{CIR}_{\text {even }}=\frac{\left|\mathrm{S}(0)+\mathrm{S}\left(\frac{\mathrm{N}}{2}\right)\right|^{2}}{\sum_{l \neq k, l=0}^{N-1}\left[S(l-k)+S\left(l-k+\frac{N}{2}\right)\right]^{2}} \\
& \mathrm{CIR}_{\mathrm{odd}}=\frac{\left|\mathrm{S}(0)-\mathrm{S}\left(\frac{\mathrm{N}}{2}\right)\right|^{2}}{\sum_{l \neq k, l=0}^{N-1}\left[S(l-k)-S\left(l-k+\frac{N}{2}\right)\right]{ }^{2}} \\
& \mathrm{CIR}_{\text {tot }}=\mathrm{CIR}_{\text {even }}+\mathrm{CIR}_{\text {odd. }}
\end{aligned}
$$

Figure 4 shows the comparison of $\mathrm{CIR}_{\mathrm{tot}}(\mathrm{in} \mathrm{dB}$ ) and $\mathrm{CIR}$ of standard OFDM as a function of normalized frequency $\epsilon$, where $\mathrm{N}=128$.CIR improvement can be found about $30 \mathrm{~dB}$.

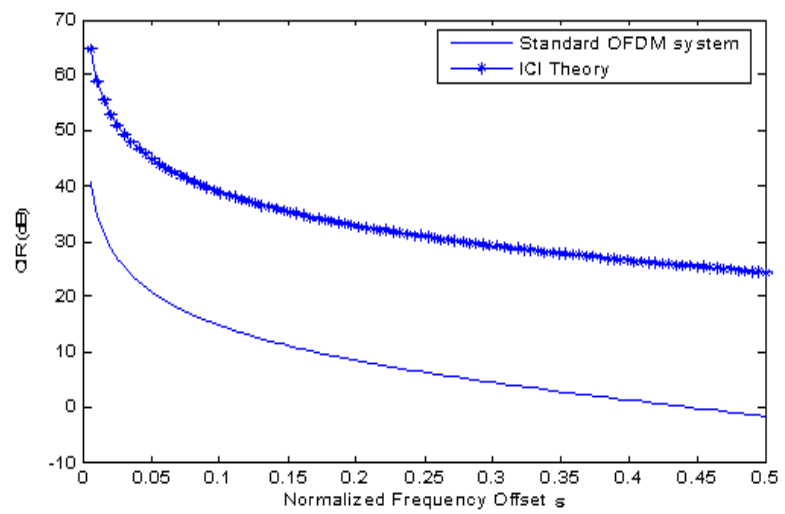

Fig 4:- Comparison of CIR v/s $\mathrm{C}$ of standard and proposed model

Performance of proposed sheme can be analyzed by BER.BER decreases as signal to noise ratio increases . Part of simulation Parameters are FFT /IFFT for 128 points, BPSK Modulation, AWGN Channel, Ns=50 Number of OFDM symbols 
Transmitted, ep $=\left[\begin{array}{lll}0.1 & 0.2 & 0.3\end{array}\right]$ carrier frequency offset, $\mathrm{E}_{\mathrm{b}} / \mathrm{N}_{0}=1: 2: 20 \quad \mathrm{SNR}$ (signal to Noise Ratio)

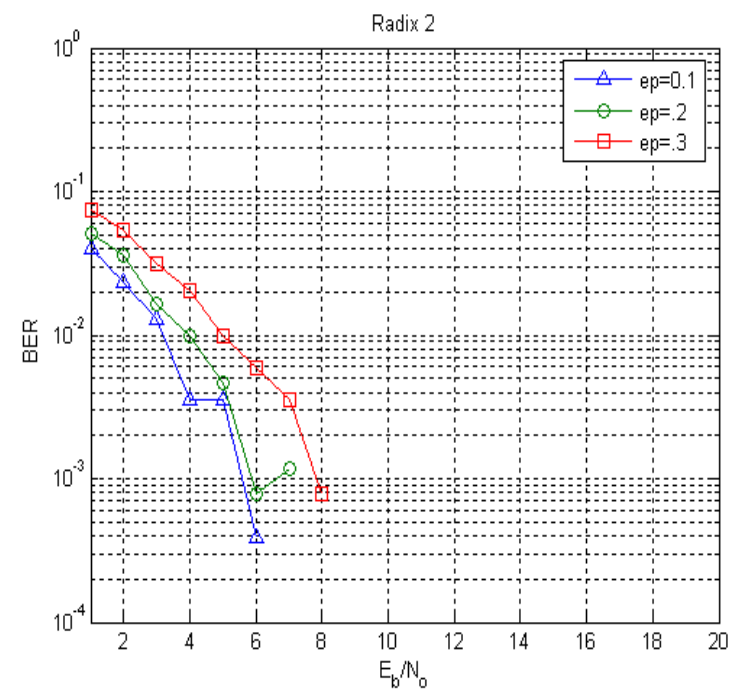

Fig 5 :- BER V/S Eb/No at ep=0.1,0.2 \&0.3

In figure 5 ,it is observed that for frequency offset 0.1 , at $\mathrm{SNR}=6 \mathrm{db}$,BER is less than $10^{-2}$.For ep=0.2,BER is slightly less than $10^{-3}$ and for ep $=0.3$,BER is greater then $10^{-4}$. So the proposed scheme shows very good performance Performance can also be judged when signal is affected by both AWGN and rician fading as below

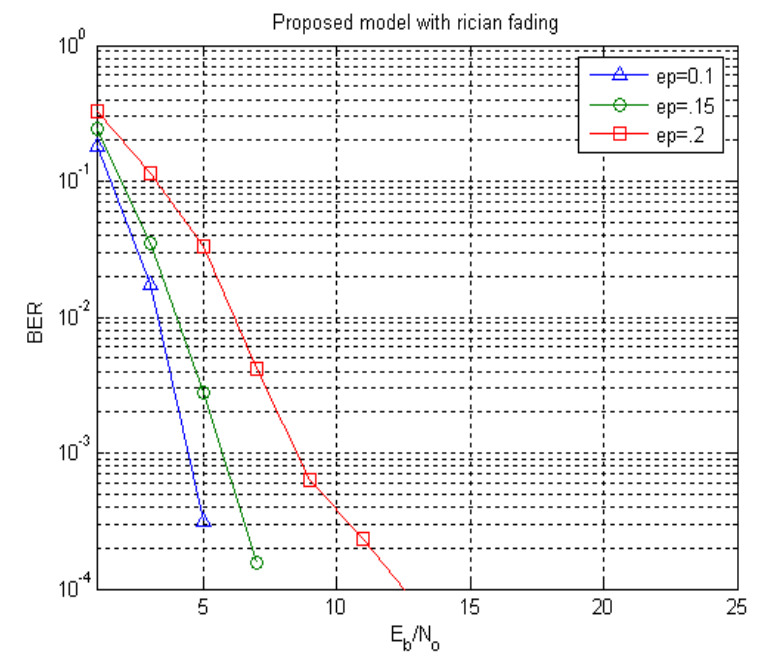

Fig 6:- BER V/S Eb/No at ep=0.1,0.15 \&0.2

In figure 6 ,it is observed that for frequency offset 0.1 , at $\mathrm{SNR}=5 \mathrm{db}, \mathrm{BER}$ is less than $10^{-3}$. For ep $=0.15, \mathrm{BER}$ is greater than $10^{-3}$ and for ep $=0.2$,BER is greater then $10^{-2}$. So the proposed scheme shows very good performance even in recian fading channel.

\section{AN IMPROVED PROPOSED MODEL}

The system performance of proposed model can also be improved by adding an extra block of convolution encoder and interleaving for forward error correction at transmitter side. At receiver, inverse of it that is convolution decoder and deinterleave is applied. Thus single or burst errors can be detected and corrected which results in more reduced BER. This can be clearer by the comparison of proposed model with convolution and without convolution. Design parameters are: FFT /IFFT for 128 points, QAM Modulation, $1 / 2$ rate Convolution coding, N/4 Length of Guard Period, AWGN Channel ,Ns=50 Number of OFDM symbols Transmitted e $\mathrm{p}=[0.2] \quad$ carrier frequency offset, $\mathrm{E}_{\mathrm{b}} / \mathrm{N}_{0}=1: 2: 20$ SNR (signal to Noise Ratio)

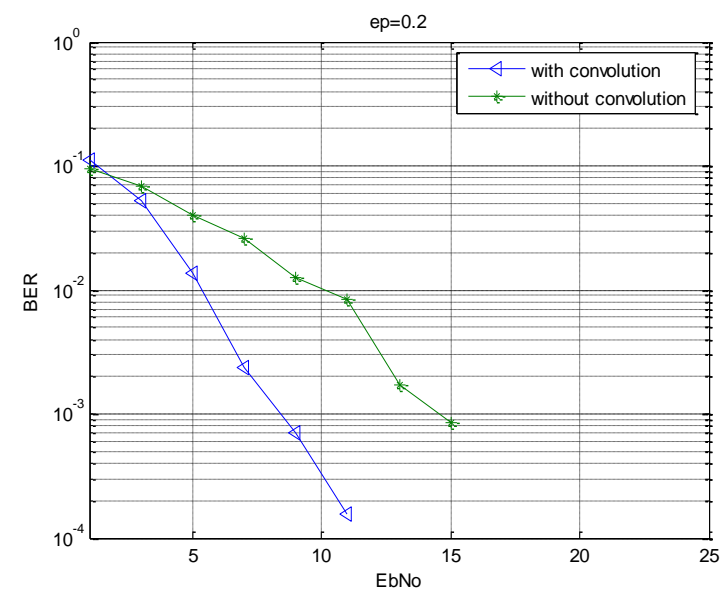

Fig 7:-Comparison B/w Proposed scheme with or without convolution

Comparison shows that convolution improves the system performance .For example, for frequency offset of 0.2, at $\mathrm{SNR}=10 \mathrm{db}, \mathrm{BER}$ of improved model is greater then $10^{-4}$ and of proposed model is $10^{-3}$

\section{COMPARISON WITH PREVIOUS MODELS}

The simulations have been conducted for performance comparison of various bandwidth efficient techniques used for ICI cancellation in OFDM system. We have performed simulations using MATLAB for BPSK/OFDM with 128 subcarriers. The normalized frequency offset is set to be $€=0.3$ respectively. The performance of various schemes of ICI cancellation is compared on basis of bit error rate (BER). We have assumed ideal reception of OFDM symbols for all schemes. BER of standard OFDM, DFT-OFDM, TPA algorithm and proposed (Radix2) algorithm is plotted in figure 8. . In figure 8 , it can be observed DFT-OFDM and Radix2 algorithm has significant improvement in performance than 
other schemes at $C=0.3$. The $\mathrm{SNR}$ gain of $10 \mathrm{~dB}, 5 \mathrm{~dB}, 13 \mathrm{~dB}$ is achieved at $10^{-2}$ for DFT-OFDM, TPA algorithm and Radix2 algorithm over standard OFDM performance. The proposed scheme outperforms than other band efficient algorithms at all frequency offsets. The spectral efficiency of proposed scheme is maintained without much increase in system complexity.

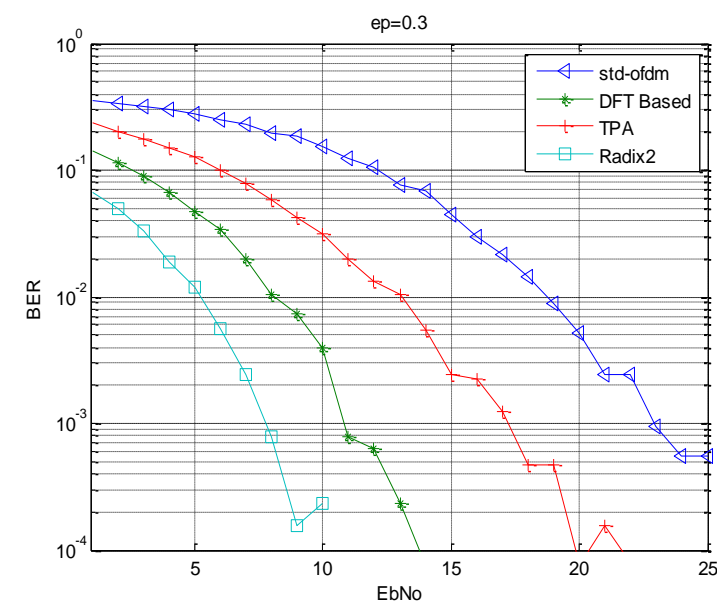

Fig8:- BER performance with Bandwidth ICI cancellation Schemes at $\mathbf{C}=0.3$

\section{CONCLUSION}

This paper basically studied the effect of ICI on OFDM system. The Proposed scheme provides significant improvement of CIR which has been studied theoretically and by simulation. The scheme works well in rician fading also. In addition, at high frequency offset it has been verified that proposed scheme is better then previous bandwidth efficient schemes like: DFTOFDM, TPA scheme without sacrificing system bandwidth efficiency.

\section{REFERENCES}

[1] Pollet T, Van Bladel M, Moeneciaey M., "BER sensitivity of OFDM system to carrier frequency offset and Wiener phase noise", IEEE Trans Commun, Vol 43,pp 191-193, 1993.

[2] J. Ahn and H. S. Lee, "Frequency domain equalization of OFDM signal over frequency nonselective Rayleigh fading channels," Electron. Lett., vol.29, No.16, pp.14761477, Aug. 1993.
[3] C. Muschallik, "Improving an OFDM reception using an adaptive Nyquist windowing," IEEE Trans. Consumer Electron., vol.42, pp. 259- 269, Aug. 1996.

[4] Y. Zhao, J.-D. Leclercq, and S.-G. Häggman, "Intercarrier interference compression in OFDM communication systems by using correlative coding," IEEE Commun. Lett., vol. 2, pp. 214-216, Aug. 1998.

[5] Y. Zhao and S-G. Haggman, "Intercarrier Interference Self-cancellation Scheme for OFDM mobile communication systems," IEEE Trans. Commun., vol.49, No.7, pp.1185-1191, July 2001.

[6] K. Sathananthan and C. Tellambura, "Probability of error calculation of OFDM systems with frequency offset," IEEE Trans. Commun., vol. 49, no. 11, pp. 1884-1888, Nov. 2001

[7] K. Sathananthan, C. R. N. Athaudage, and B. Qiu, "A novel ICI cancellation scheme to reduce both frequency offset and IQ imbalance effects in OFDM," in Proc. IEEE 9th International Symposium on Computers and Communications, pp. 708-713, July 2004.

[8] Bing Han, Xiqi Gao et al., "A DFT-based ICI Selfcancellation Scheme for OFDM Systems," Commun. Technology Proc., ICCT, vol. 2, pp. 1359-1362, April 2003.

[9] Heung-GyoonRyu, Member, IEEE, Yingshan Li, and JinSoo Park, Member, IEEE “An Improved ICI Reduction Method in OFDM Communication System" IEEE TRANSACTIONS ON BROADCASTING, VOL. 51, NO. 3, SEPTEMBER 2005

[10] Arvind Kumar, and Rajoo Pandey," A bandwidth-efficient method for cancellation of ICI in OFDM systems," Int. J. Electron.Communication., volume 63, pp 569-575, 2009

[11] Akhil Kamboj, Abhinav Keshari, Vivek K. Dwivedi1, and G. Singh, "Bandwidth Efficient Intercarrier Interference Cancellation Technique for OFDM Digital Communication Systems", PIERS Proceedings, Vol 5, pp 1244-1248, August 2009.

[12] Qiang Shi, Yong Fang and Min Wang, "A Novel ICI Self Cancellation Scheme for OFDM Systems", 5th International Conference on Wireless Communications Networking and Mobile Computing, pp. 1-4. October 2009.

[13] Fan JunLe, Hu YongJiang, Chen ZiLi "A New ICI Self Cancellation Method with Double Bandwidth Efficiency in OFDM Systems" 2010 International Conference on Communications and Mobile Computing. 